\title{
L'ordre public et le droit de la liberté de religion en Allemagne
}

Claus Dieter Classen

\section{(2) OpenEdition}

\section{Journals}

Édition électronique

URL : http://journals.openedition.org/rdr/1117

DOI : $10.4000 /$ rdr. 1117

ISSN : 2534-7462

Éditeur

Presses universitaires de Strasbourg

\section{Édition imprimée}

Date de publication : 9 juillet 2020

Pagination : 73-89

ISBN : 979-10-344-0065-2

ISSN : 2493-8637

Référence électronique

Claus Dieter Classen, «L'ordre public et le droit de la liberté de religion en Allemagne », Revue du droit des religions [En ligne], 9| 2020, mis en ligne le 18 mai 2020, consulté le 20 novembre 2020. URL : http://journals.openedition.org/rdr/1117 ; DOI : https://doi.org/10.4000/rdr.1117

\section{(c) $(1)(9$}

La revue du droit des religions est mise à disposition selon les termes de la Creative Commons Attribution - Pas d'Utilisation Commerciale 4.0 International - CC BY-NC 4.0. 


\section{L'ORDREPUBLICET LEDROITDELALIBERTÉ DERELIGION ENALLEMAGNE}

\section{Claus Dieter CLASSEN}

Universität Greifswald

\section{RÉSUMÉ}

En Allemagne, les exigences constitutionnelles permettant de limiter la liberté de religion afin de protéger l'ordre public sont bien plus sévères qu'en France. Seules les mesures qui sont justifiées par une valeur ancrée dans la Constitution sont acceptées. Ainsi, tous les recours constitutionnels individuels fondés sur une violation de la liberté de religion ont jusqu'ici été couronnés de succès, et on ne trouve guère d'exemples où l'ordre public prime la liberté de religion, y compris dans la jurisprudence des autres tribunaux.

\section{Abstract}

In Germany the freedom of religion is protected much better than in France. It can only be restricted if this is justified by a value enshrined in the Constitution itself. Thus, all constitutional remedies based on a violation of freedom of religion have so far been successful, and there are very few examples where public order overweighs the freedom of religion in the case law of other courts. 


\section{INTRODUCTION}

La liberté de religion est un des droits fondamentaux de l'homme. Rien d'étonnant donc à ce qu'elle soit inscrite à l'article 4 de la Constitution allemande. Dans les États sécularisés, la liberté de croire en ce qu'on estime être juste ou vrai ne pose pas de problème pour la coexistence des individus. Mais la quasi-totalité des religions dans le monde ne se contentent pas d'une démarche intellectuelle. Elles se fondent au contraire sur une certaine représentation du monde et de ce que doivent être les comportements humains sur terre. Elles comprennent ainsi souvent des prescriptions relatives à la vie des personnes. Par conséquent, il est communément admis que la liberté de religion, telle qu'elle est énoncée dans la Constitution allemande, protège également le droit pour une personne «d'adapter la totalité de son comportement aux dogmes de sa religion et d'agir conformément à ses convictions religieuses intimes ${ }^{1} »$.

Mais cette liberté peut également entrer en conflit avec d'autres droits fondamentaux. La question se pose alors de savoir comment résoudre de tels conflits. En France, c'est le concept fondamental d'ordre public qui permet de poser des limites à la liberté de religion. En Allemagne, la situation juridique est plus complexe. L'objet des développements qui suivent est d'examiner les questions qui en découlent. Il conviendra dans un premier temps d'évoquer les fondements juridiques pertinents, avant d'étudier plus en détail l'application concrète de ces principes dans des cas spécifiques.

\section{FONDEMENTS JURIDIQUES}

\subsection{L'ORDRE PUBLIC}

En France, la notion d'ordre public joue un rôle prépondérant en droit de la police. En droit allemand, l'ordre public est également reconnu comme un concept pertinent par les lois de police de tous les Länder. Il ne joue cependant qu'un rôle très limité, car il est situé au même niveau que la notion de sécurité publique, à laquelle il est la plupart du temps fait référence en pratique. Par sécurité publique, on entend l'inviolabilité de l'ordre juridique, l'inviolabilité des établissements et des activités de l'État ainsi que

1. Décision de la Cour constitutionnelle fédérale BVerfGE 32, 98 (106), 19-10-1971; 33, 23 (28 et s.), 11-04-1972. 
l'inviolabilité des droits fondamentaux des individus. L'ordre public quant à lui comprend l'ensemble des règles de comportement non écrites, dont le respect, selon la doctrine dominante, est la condition sine qua non d'un vivre ensemble harmonieux ${ }^{2}$.

\subsection{LIBERTÉ DE RELIGION ET ORDRE PUBLIC}

Ainsi qu'il vient d'être dit, la Constitution allemande protège la liberté de religion en son article 4. Étant donné que celle-ci est comme en France susceptible d'entrer en conflit avec d'autres principes fondamentaux, la question est posée de savoir dans quelles conditions il est possible de la restreindre. Dans cette hypothèse, on se trouve confronté au problème suivant: l'article 4 de la Loi fondamentale allemande ainsi que certains autres droits fondamentaux, ce qui est le cas des libertés garanties de l'art et de la science (art. 5 al. 3), ne contiennent aucune réserve expresse autorisant une restriction légale, contrairement à la plupart des autres droits fondamentaux. Dans les années 1960 et 1970, il a été longuement débattu des conséquences de cette situation juridique. La solution consistant à emprunter les restrictions fixées pour d'autres droits fondamentaux a été rejetée, de même que celle consistant à n'en fixer aucune. La première approche aurait clairement transgressé les prescriptions constitutionnelles non équivoques établissant une réglementation limitative particulière à chacun des droits fondamentaux. La deuxième approche quant à elle aurait accordé une trop grande importance à la liberté de religion. Il a donc été décidé d'adopter une voie intermédiaire. Depuis le début des années 1970, il est largement admis que les interventions dans le champ des droits fondamentaux non assortis de limites explicites, et par conséquent dans celui de la liberté de religion, ne sauraient être admises pour la protection de n'importe quel intérêt public légitime. La condition d'une telle limitation est qu'elle assure la protection de valeurs ancrées dans la Constitution, notamment d'autres droits fondamentaux protégés par la Constitution ${ }^{3}$. L'idée qui a présidé à cette réflexion est que la Constitution dans son ensemble forme une unité. Un droit garanti par la Constitution ne peut donc pas être interprété de telle manière que d'autres droits soient entravés plus que de raison; il faut garantir entre eux un «soigneux équilibre ${ }^{4} »$.

2. Sur les deux points, BVerfGE 69, 315 (352), 14-05-1985.

3. BVerfGE 28, 243 (260 et s.), 26-05-1970, à propos du droit à l'objection de conscience (art. 4 al. 3 de la Loi fondamentale). BVerfGE 30, 173 (192 et s.), 24-02-1971 à propos de la liberté de l'art (art. 5 al. 3 de la Loi fondamentale).

4. P. Lerche, Übermaß und Verfassungsrecht, Köln, Heymann, $2^{e}$ éd. 1999, p. 153. 
Dans la mesure où les droits des tiers ainsi que l'existence de l'État sont en cause, il est toujours possible de recourir à des normes constitutionnelles permettant de légitimer une intervention. L'ordre juridique en tant que tel, l'ordre public du point de vue français et à plus forte raison l'ordre public tel qu'on l'entend en Allemagne, ne permet pas en revanche de justifier de telles restrictions. La liberté de religion - tout comme la liberté de l'art - peut être qualifiée de «résistante à la police»: ni la police ni aucune autre autorité étatique ne peuvent interférer dans ces libertés dans les conditions généralement applicables, mais uniquement si les conditions spécifiques précitées sont remplies, c'est-à-dire s'il s'agit de protéger un droit fondamental ancré dans la Constitution. Simultanément, il est clair que la liberté de religion jouit ainsi d'une protection qui dépasse nettement celle accordée à la plupart des autres droits fondamentaux. Le principe, énoncé par le Conseil constitutionnel français, selon lequel une personne ne peut «se prévaloir de ses croyances religieuses pour s'affranchir des règles communes régissant les relations entre collectivités publiques et particuliers ${ }^{5} »$ ne trouve donc pas à s'appliquer en Allemagne.

\subsection{CONDITIONS REQUISES POUR LA PROTECTION DE LA LIBERTÉ DE RELIGION}

La protection spécifique de la liberté de religion ne peut être garantie qu'à la condition que celle-ci trouve à s'appliquer. Toutefois, l'article 4 de la Loi fondamentale ne mentionne que certains aspects de la liberté de religion, à savoir la liberté de croyance, la liberté de confession et la liberté de pratiquer une religion. Concrètement, la liberté de croyance protège la conviction intime d'une personne (liberté du for interne), la liberté de confession protège l'expression et la diffusion de la croyance et la liberté de culte protège l'ensemble des pratiques rituelles ${ }^{6}$.

Mais la jurisprudence de la Cour constitutionnelle fédérale va, et ce depuis longtemps, encore plus loin. La liberté de religion protège également l'ensemble des comportements humains qui ont un fondement religieux, en particulier s'ils sont dictés par les règles de conduite de la religion de la personne concernée. La Cour constitutionnelle souligne ainsi que l'article 4 ne protège pas seulement l'accomplissement des prescriptions religieuses obligatoires, mais la totalité des actes, y compris s'ils sont uniquement guidés par la croyance ${ }^{7}$.

5. Cons. const., 19 nov. 2004, no 2004-505 DC, \& 18.

6. Détails chez C. D. Classen, Religionsrecht, Tübingen, Mohr Siebeck, 2. Aufl. 2015, no 146 et $s$.

7. V. réf. de la note 1. 
Il n'est cependant pas rare que des différends surgissent quant à savoir si un acte est effectivement lié à la croyance. Les débats à propos du voile islamique en sont un exemple parmi d'autres. La Cour constitutionnelle allemande se montre régulièrement compréhensive dans ce domaine: c'est la représentation que la personne concernée a d'elle-même qui est ici déterminante. Si cette dernière explique de façon plausible en quoi un comportement précis est important pour elle pour des raisons religieuses, la juridiction étatique doit l'accepter, sans pouvoir le contrôler et encore moins porter un jugement de valeur ${ }^{8}$.

D'un point de vue individuel, la liberté de religion protège en premier lieu le croyant. Mais en vertu de l'article 19 alinéa 3 de la Constitution allemande, «les droits fondamentaux s'appliquent également aux personnes morales nationales lorsque leur nature le permet». Les personnes morales en tant que telles n'ont pas de croyance, mais la religion se pratique de façon régulière dans le cadre de communautés. Il en résulte donc que les communautés religieuses peuvent également se prévaloir de la liberté de religion. Mais il existe une spécificité dans ce domaine. Le droit à l'autoadministration garanti par l'article 137 alinéa 3 de la Constitution de Weimar bénéficie également aux communautés religieuses, dans la mesure où cette disposition fait partie intégrante de la Constitution allemande en application de l'article 140 de celle-ci. Ce droit s'étend au-delà de la liberté de religion, dans la mesure où il inclut non seulement les matières religieuses, mais aussi toutes les actions entreprises par la communauté religieuse. Toutefois ce droit à l'autodétermination est soumis - bien qu'elle soit restreinte - à une réserve de loi, à savoir qu'il s'exerce dans «les limites de la loi applicable à tous» (voir de plus amples détails à ce sujet au point 4.). La protection de la liberté de religion des groupements religieux ne s'étend cependant pas au-delà de la liberté garantie dans le cadre de l'article 137 alinéa 3 de la Constitution de Weimar ${ }^{9}$.

En fin de compte, la liberté de religion ne déploie sa protection de façon illimitée que dans les cas d'«ingérence» dans l'exercice de cette liberté. Par ingérence, on entend toutes les interventions étatiques qui restreignent l'espace de liberté décrit ci-dessus. Il ne s'agit pas nécessairement de mesures juridiques proprement dites. Même des mesures purement factuelles, comme

8. Spécialement concernant le voile islamique: BVerfGE 108, 282 (298), 24-09-2003; V. également BVerfGE 24, 236 (247 et s.), 16-10-1968 (au III.1.a) ainsi que BVerfGE 33, 23 (29 et s.), 11-04-1972 (au III.4.b).

9. BVerfGE 137, 273 (85), 22-10-2014. 
le fait d'accrocher un crucifix dans une école ${ }^{10}$ ou des critiques émanant de services étatiques ${ }^{11}$, peuvent constituer des ingérences.

\subsection{CONDITIONS DE JUSTIFICATION DES INGÉRENCES DANS L'EXERCICE DE LA LIBERTÉ DE RELIGION}

Ainsi qu'il a été dit, une ingérence dans la liberté de religion peut être justifiée. Mais il faut que certaines conditions soient remplies. La condition la plus importante a déjà été mentionnée, à savoir que l'ingérence doit avoir pour objet la protection de valeurs inscrites dans la Constitution. Mais elle ne peut avoir pour seul fondement une disposition constitutionnelle. Elle doit également trouver sa justification dans une loi ordinaire rédigée de façon suffisamment concrète.

Une ingérence dans le champ de la liberté religieuse doit également être proportionnée. Elle doit être adaptée pour atteindre l'objectif poursuivi. Elle doit être le moyen le moins contraignant pour atteindre l'objectif recherché avec la même efficacité. Enfin, elle doit être adéquate. Ce qui est en cause ici, c'est l'importance des intérêts juridiquement protégés en présence rapportée à l'intensité de l'ingérence ${ }^{12}$. La Cour constitutionnelle allemande souligne régulièrement qu'en matière de liberté de religion, l'appréciation de la personne concernée joue un rôle déterminant.

Comme on l'a dit, il existe des particularités propres aux communautés religieuses. En ce qui les concerne, c'est la loi commune applicable à tous qui fixe les limites. Quant à savoir si une loi est effectivement «applicable à tous ", la Cour constitutionnelle fédérale ne s'intéresse pas seulement à la façon dont elle est formulée. C'est son contenu matériel qui importe. Concrètement, cela signifie qu'une loi ne s'applique «à tous» dans le sens compris ci-dessus que si elle concerne matériellement la communauté religieuse au même titre que n'importe quelle organisation ${ }^{13}$. Si en revanche la communauté religieuse est plus concernée que d'autres en raison de ses spécificités religieuses, la réserve des limites imposées ne s'applique pas. Dans ce cas, la mesure en cause doit trouver son fondement dans un des droits fondamentaux de la Constitution, comme dans le cas de la liberté individuelle de religion.

10. BVerfGE 93, 1 (17 et s.), 16-05-1995.

11. BVerfGE 105, 279 (300 et s.), 26-05-2002.

12. Pour cette structure, V. T. Hochmann, «Droits fondamentaux», in A. Gaillet et. al., Droits constitutionnels français et allemand, Paris, LGDJ, 2019, $\mathrm{n}^{\circ} 724$ et s.

13. BVerfGE 42, 312 (344), 21-09-1976. 


\subsection{LA LIBERTÉ DE RELIGION EN DROIT PRIVÉ}

Par souci d'exhaustivité, il convient de préciser que les considérations qui précèdent ne valent que pour les mesures étatiques. Mais la liberté de religion n'est pas pour autant dépourvue de signification en droit privé. En effet, elle fait partie du système de valeurs des droits fondamentaux, et en tant que telle elle doit être protégée par l'État. En conséquence, le juge doit tenir compte de façon appropriée de la liberté de religion lorsqu'il interprète une règle de droit ordinaire, c'est-à-dire qu'il doit également prendre en considération les droits fondamentaux de l'autre partie. Cela a des conséquences pratiques, notamment en droit du travail.

\section{L'APPLICATION DE CE PRINCIPE DANS LA PRATIQUE}

Si l'on s'interroge en détail sur l'importance de la liberté de religion dans les conflits avec d'autres droits fondamentaux, il y a lieu de distinguer les cas où le conflit relève de l'ordre juridique général, et ceux où la partie concernée entretient une relation particulière avec l'État, ce qui en France correspond au champ du service public.

\subsection{LA LIBERTÉ DE RELIGION INDIVIDUELLE ET L'ORDRE JURIDIQUE GÉNÉRAL}

\subsubsection{L'AFFAIRE DU CHIFFONNIER}

En 1965, une organisation de jeunesse catholique avait organisé une «opération de débarras». Dans le cadre de cette action, l'organisation a collecté des vêtements et des chiffons usagés ainsi que du vieux papier pour les vendre à des grossistes afin d'utiliser les fonds récoltés pour des projets d'aide au développement. Cette opération a été notamment annoncée lors des prêches dans les églises catholiques. Suite à cette action, un revendeur de matières premières a été confronté à de graves difficultés économiques, car il ne recevait quasiment plus de vieux chiffons et n'avait pratiquement plus rien à vendre. Il a donc porté plainte contre l'organisation de jeunesse catholique pour obtenir des dommages et intérêts et l'interruption de l'activité. Il a obtenu gain de cause parce que le tribunal a classé cette action comme immorale et donc comme faussant la concurrence aux termes du $\S 1$ de la loi contre la concurrence déloyale. 
Le recours constitutionnel individuel (Verfassungsbeschwerde, ci-après recours constitutionnel) exercé contre cette décision a été un succès ${ }^{14}$. Selon la Cour constitutionnelle fédérale, il n'avait pas été suffisamment tenu compte de la liberté de religion dans l'interprétation et l'application de la notion d'«immoralité». La notion de pratique religieuse qui comprend toutes les «formes d'expression de la vie religieuse » doit être interprétée au sens large en raison de son importance majeure pour la croyance en cause. Quant à savoir ce que cette notion inclut concrètement, il faut tenir compte de la propre perception (auto-compréhension) de la communauté religieuse concernée. Selon l'auto-compréhension de l'Église catholique, la pratique religieuse comprend également le développement et l'engagement dans le monde, en particulier sous la forme d'actions caritatives. Les collectes de dons en font également partie dès lors que la générosité des donateurs est fondée sur des motifs religieux manifestes.

La Cour ne se penche que brièvement sur la question de savoir si la décision judiciaire d'interdiction est conforme à la Constitution: les activités de la jeunesse catholique font partie de la pratique religieuse régulière et elles n'auraient donc pas dû être qualifiées d'«immorales».

\subsubsection{L'AFFAIRE DU PRÊCHEUR-GUÉRISSEUR}

Un autre cas concernait les Témoins de Jéhovah qui, comme on le sait, refusent les transfusions sanguines. Une femme qui aurait eu besoin d'une transfusion a refusé de se faire hospitaliser, en accord avec son mari et contre l'avis des médecins, pour ne pas avoir à subir une telle transfusion. Elle a prié avec son mari, mais cela seul n'a pas suffi. Après le décès de la femme, consciente jusqu'à la fin, l'homme a été condamné pour non-assistance à personne en danger.

La Cour constitutionnelle fédérale ${ }^{15}$ a dans un premier temps souligné que la liberté de religion garantit «à l'individu un espace juridique libre de toute ingérence de l'État». Cet espace de liberté «comprend également le droit pour chaque individu de conformer en tout sa conduite aux dogmes de sa foi et d'agir conformément à ses convictions religieuses intimes». Cette liberté est certes soumise à des limites. Toutefois, la signification particulière de la liberté de religion exclut «d'exposer sans discernement les activités et comportements résultant d'une attitude de foi aux sanctions que l'État prévoit

14. BVerfGE 24, 236, 16-10-1968.

15. BVerfGE 32, 98, 19-10-1971. 
en de telles situations en dehors de toute motivation religieuse». Dans le cas présent, il convient donc de se demander si une sanction répondrait encore à la définition d'une peine étatique dans la mesure où son auteur ne se rebelle pas contre l'ordre juridique étatique par défaut d'éthique juridique. En tout état de cause, le recours au droit pénal comme arme la plus affûtée au service de la société n'était pas approprié dans le cas présent. Sur le plan pénal, il «est impossible d'exiger que deux personnes, partageant les mêmes sentiments religieux, s'influencent mutuellement pour se convaincre du danger d'une décision résultant de leur croyance commune».

\subsubsection{LE PORT D'UN CASQUE DE MOTO PAR LES SIKHS}

On peine à trouver des décisions judiciaires dans lesquelles le conflit entre la liberté de religion et d'autres intérêts juridiquement protégés a été tranché au détriment de la liberté religieuse. On peut toutefois mentionner une décision du Tribunal administratif fédéral concernant l'obligation faite aux conducteurs de motocyclettes de porter un casque. Un sikh obligé par sa religion de porter un turban a demandé une exception, sans succès. Certes, les dispositions applicables prévoient une telle dispense. De plus, les juges du fond lui avaient donné raison dans la mesure où ils avaient obligé l'administration compétente à reconsidérer sa demande, car celle-ci n'avait pas pris en considération la possibilité d'accorder une telle dérogation en raison de la religion du demandeur. Cependant, la demande tendant à imposer directement à l'Administration d'accorder une telle exception a été rejetée. L'ingérence dans la liberté religieuse contenue dans cette obligation se justifie par la nécessité de protéger les autres personnes en cas d'accident: elles peuvent être traumatisées par la vue d'un motard blessé. Un motard peut en outre plus facilement porter secours à d'autres personnes s'il porte un casque ${ }^{16}$.

\subsubsection{LA CIRCONCISION DES ENFANTS}

Il n'existe à ce jour aucune jurisprudence de la Cour constitutionnelle ni du Tribunal administratif fédéral sur la question de la circoncision. Il convient néanmoins de citer une décision du tribunal de grande instance de Cologne datant de 2012, où la question posée était de savoir si le droit qu'ont les parents de définir l'intérêt de leurs enfants justifie la décision de faire circoncire leur garçon. Les juges ont répondu par la négative, dans une affaire pénale où la circoncision pratiquée avait entraîné des complications 
médicales. En tout état de cause, le tribunal est ici parti du principe qu'une telle décision était prohibée de façon absolue parce qu'elle constituait une atteinte illégale à l'intégrité physique ${ }^{17}$. Cette décision a provoqué un débat animé, à la fois parmi les experts et dans l'opinion publique ${ }^{18}$. Dans l'affaire en cause, les parents étaient musulmans. Mais quoi qu'il en soit, il était manifeste que le raisonnement juridique suivi interdisait également de fait aux juifs de se conformer à un commandement pourtant constitutif de leur identité. Le législateur est donc intervenu et a reconnu explicitement le droit pour les parents de circoncire leur enfant ( $\$ 1631 \mathrm{~d}$ du Code civil allemand).

\subsection{LA LIBERTÉ DE RELIGION DES COMMUNAUTÉS RELIGIEUSES}

\subsubsection{L'ABATTAGE RITUEL}

Le litige concernant l'abattage rituel des animaux constitue un autre exemple. Tout comme en France, la loi allemande sur la protection des animaux interdit par principe de telles pratiques d'abattage. Elles sont cependant autorisées à titre exceptionnel, dans la mesure où elles sont nécessaires pour « répondre aux besoins de membres de certaines communautés religieuses [...], auxquels les prescriptions obligatoires de leur communauté religieuse imposent l'abattage rituel des animaux ou interdisent la consommation de viande d'animaux qui n'ont pas été abattus rituellement» (§ 4a Tierschutzg$z s e t z$ - loi allemande relative à la protection des animaux). Mais lorsqu'une communauté musulmane a demandé une autorisation d'abattage rituel, cette autorisation lui a été refusée. Pour justifier cette décision, le Tribunal administratif fédéral a précisé, en référence à la loi relative à la protection des animaux, que les règles régissant la communauté n'étaient pas suffisamment claires et ne pouvaient de ce fait bénéficier de l'exception mentionnée cidessus. De plus, le tribunal n'a pas vu dans le litige une question liée à la liberté de religion. La consommation de viande n'étant pas protégée au titre de celle-ci ${ }^{19}$, chacun peut en effet y renoncer de lui-même.

La Cour constitutionnelle fédérale, saisie du recours d'un boucher musulman, a quant à elle décidé que le Tribunal administratif fédéral avait mal apprécié l'impact de la liberté constitutionnelle de religion sur la loi

17. Landgericht Köln (tribunal de grande instance de Cologne) : JZ 2012, p. 805.

18. Du même avis que le tribunal d'instance, V. par ex. J. ISENSEE, «Grundrechtliche Konsequenz wider geheiligte Tradition», JZ 2013, p. 317; pour un avis critique, T. HörNLE, S. Huster, «Wie weit reicht das Erziehungsrecht der Eltern?», JZ 2013, p. 328.

19. BVerwGE 99, 1 (7 et s.), 15-06-1995. 
relative à la protection des animaux ${ }^{20}$. Compte tenu d'une part des habitudes alimentaires allemandes et d'autre part des convictions religieuses des personnes concernées, le fait d'entraver de façon manifeste l'approvisionnement en viande issue de l'abattage rituel constitue une atteinte à la liberté de religion. La dérogation prévue par la loi sur la protection des animaux n'impose pas l'existence d'une communauté religieuse formellement structurée, mais se satisfait d'un groupe de personnes partageant la même conviction religieuse. Ce qui était le cas en l'occurrence. Quant à savoir si, dans cette affaire, la dérogation prévue par la loi sur la protection des animaux était également nécessaire, la Cour constitutionnelle fédérale ne s'est pas prononcée.

\subsubsection{INTERDICTION D'ENTRÉE SUR LE TERRITOIRE POUR LE CHEF SPIRITUEL DU MOUVEMENT MOON}

Un recours en inconstitutionnalité des adeptes allemands de «l'Église coréenne de l'unification» (communauté Moon) a également été couronné de succès ${ }^{21}$. Dans le cadre d'une tournée mondiale, son chef spirituel devait se rendre en Allemagne. Les autorités allemandes s'y sont opposées, et ont notifié aux époux Moon un refus d'entrée sur le fondement de la Convention d'application des accords de Schengen, attaqué en vain devant le Tribunal administratif fédéral ${ }^{22}$. Ne tenant aucun compte de la liberté de religion, les tribunaux avaient en effet jugé le recours irrecevable. Compte tenu de ses modalités concrètes, le voyage ne présentait pas selon eux un caractère religieux spécifique.

En réponse, la Cour constitutionnelle fédérale souligne que la liberté de religion ne confère bien entendu aucun droit à l'entrée sur le territoire. En l'espèce toutefois, les conditions requises pour refuser l'entrée sur le territoire allemand n'avaient pas fait l'objet d'un examen détaillé, car les tribunaux avaient mal apprécié l'importance que présente sur le plan religieux, pour les adeptes allemands, la visite des époux Moon dans leur pays. La liberté de religion avait en conséquence été méconnue dans la mesure où, du point de vue de la liberté de religion, une visite du chef spirituel de la communauté Moon revêt une importance fondamentale pour ses adeptes. La Cour constitutionnelle fédérale ne s'est pas prononcée en revanche sur la question de savoir si le couple aurait pu obtenir un droit d'entrée sur le territoire allemand.

20. BVerfGE 104, 337 (350), 15-01-2002.

21. BVerfG (chambre), 2 BvR 1908/03, 24-10-2006.

22. BVerwG, 1 B 288.02, 04-09-2003. 


\subsubsection{INHUMATION DANS UNE CRYPTE}

Le recours constitutionnel d'une paroisse syrienne orthodoxe contre le refus d'une dérogation aux fins de construction d'un lieu de sépulture pour son chef spirituel dans la crypte de son église a également été couronné de succès $^{23}$. La communauté avait avancé que, selon la sainte tradition sacrée de l'Église syrienne orthodoxe, les prêtres et eux seuls doivent reposer dans un espace particulier à l'intérieur de l'église. Une crypte pour les serviteurs de l'autel, à portée de voix de l'autel où ils ont officié, faisait ainsi partie intégrante d'un édifice religieux qui se voulait fidèle à l'authentique tradition syro-antiochienne.

L'autorité compétente a refusé parce que l'église était située dans une zone industrielle. Il lui paraissait également impossible de déroger - ce que permet en principe le droit allemand - aux dispositions contraires de la loi sur l'urbanisme. À ses yeux, les prescriptions religieuses en matière d'inhumation ne présentaient aucun caractère obligatoire. De plus, la protection post-mortem de la personnalité, le repos des morts ainsi que le sentiment de piété des proches et de la communauté des fidèles s'opposent à une telle dérogation. La paroisse a également été déboutée par les tribunaux administratifs saisis du recours.

La Cour constitutionnelle fédérale a souligné pour sa part que les références à la protection de la personnalité post-mortem, au repos des morts et au sentiment de piété des personnes endeuillées n'étaient pas pertinentes et, qu'en cas de doute, il aurait fallu tenir compte de la volonté des ecclésiastiques d'être enterrés dans l'église. En revanche, si la référence aux droits fondamentaux des entreprises industrielles environnantes - qui craignaient des restrictions d'utilisation destinées à protéger un éventuel lieu de sépulture, notamment en matière de protection sonore - devait être prise au sérieux, il reste que le tribunal n'avait pas suffisamment pris en considération la liberté de religion. Il en allait de même s'agissant de l'offre faite par la communauté religieuse de prendre elle-même des mesures pour résoudre le conflit.

\subsubsection{LA DÉCISION OSHO}

Un recours constitutionnel du mouvement Osho, fondé par Rajneesh Chandra Mohan, à l'encontre de plusieurs déclarations du gouvernement

23. BVerfG (chambre), 1 BvR 2202/13, 09-05-2016. 
allemand, a été partiellement couronné de succès. Dans un rapport au Bundestag et dans des réponses aux questions des députés du Bundestag, le gouvernement avait notamment qualifié la partie plaignante de «religion pour les jeunes», de «secte pour les jeunes» et de «secte psychologique» qui «manipule» ses membres, en y ajoutant les adjectifs de «destructive» et de «pseudo religieuse».

De l'avis de la Cour constitutionnelle fédérale ${ }^{24}$, l'utilisation des substantifs mentionnés était licite dans la mesure où ils ne comprennent aucun qualificatif négatif. Tel n'était pas le cas en revanche s'agissant des autres déclarations. Le mouvement Osho devrait en fait être qualifié de Weltanschauung (conception du monde), une terminologie qui, en Allemagne, désigne des mouvements spirituels qui fondamentalement se présentent comme une religion mais qui ne se réfèrent à aucune transcendance. Leur liberté est protégée par les alinéas 1 et 2 de l'article 4 de la Loi fondamentale au même titre que la liberté des religions. Le gouvernement allemand a de ce fait porté une atteinte disproportionnée à l'exercice de cette liberté.

\subsubsection{LE DROIT D'ASILE DANS LES ÉGLISES}

L'asile religieux dans les églises, ainsi qu'on le désigne communément, est l'objet d'un contentieux récurrent qui a pris une importance particulière ces dernières années. Il s'agit d'une pratique consistant pour des paroisses à accueillir dans les locaux de l'église des étrangers menacés d'expulsion en vue de leur offrir une protection. Généralement informées de l'asile accordé par la paroisse, les autorités chargées de l'immigration renoncent dans la plupart des cas à une évacuation par la force.

À ce jour, ni la Cour constitutionnelle fédérale, ni la Cour fédérale, ni le Tribunal administratif fédéral n'ont encore statué sur la question de savoir si l'asile dans les lieux de culte est protégé au titre de la liberté de religion, en tant qu'expression de la charité chrétienne mise en pratique, et si et dans quelle mesure les règles étatiques relatives à l'octroi et surtout au refus de l'asile fixent des restrictions appropriées. Dans la doctrine, les avis sont partagés ${ }^{25}$.

24. BVerfGE 105, 279, 26-06-2002.

25. Pour une protection par la liberté de religion, V. M.-E. GEIS, «Kirchenasyl im demokratischen Rechtsstaat», JZ 1997, p. 60; G. RobBERS, "Kirchliches Asylrecht? », AöR 113 (1988), p. 30. Contra: V. I. von MüNCH, «"Kirchenasyl" - ehrenwert, aber kein Recht », NJW 1995, p. 565. 


\subsection{COMPLÉMENT : LA LIBERTÉ DE RELIGION DANS LE CONTEXTE DU SERVICE PUBLIC}

L'étendue de la liberté de religion au sein des services publics ne prend pas non plus en compte, selon l'interprétation allemande, la sécurité générale et l'ordre public général. Il convient néanmoins de présenter un aperçu de la jurisprudence de la Cour constitutionnelle fédérale. Elle atteste une fois de plus de l'importance accordée en Allemagne à la liberté de religion dans les conflits avec d'autres intérêts juridiquement protégés, par comparaison avec la France.

\subsubsection{LA LIBERTÉ DE RELIGION À L'ÉCOLE}

La législation scolaire fournit deux exemples d'affaires où les règles juridiques applicables n'ont pas été jugées suffisamment concrètes pour justifier des mesures constitutives d'une ingérence dans la liberté de religion. La première affaire concernait les crucifix, qui autrefois - et encore de nos jours - étaient accrochés dans les salles de classe en Bavière, sans aucun fondement légal formel. Selon la Cour constitutionnelle fédérale, un tel fondement est pourtant nécessaire, car le fait d'accrocher des crucifix dans une salle de classe constitue une ingérence dans la liberté négative de religion dépourvue de base légale. Celle-ci a été adoptée après la décision de la Cour. Les questions juridiques substantielles soulevées par cette affaire n'ont ainsi reçu qu'une réponse partiellement convaincante.

La deuxième affaire portait sur la question de savoir si une enseignante d'une école publique est en droit de porter un voile islamique. Dans une décision datant de 2008, la Cour constitutionnelle fédérale a considéré que, compte tenu de la gravité de l'atteinte aux droits fondamentaux, un fondement légal explicite était indispensable pour justifier une interdiction. Il ne suffisait pas d'invoquer l'obligation générale de neutralité des fonctionnaires, notamment parce que comme tout le monde pouvait le constater, la décision de porter le voile islamique avait été prise individuellement par une enseignante et non par un organisme gouvernemental. Néanmoins, la Cour a estimé que le simple risque abstrait de perturbation de la paix dans les écoles pouvait servir de fondement à une interdiction légale ${ }^{26}$.

Sur ce dernier point, la Cour constitutionnelle fédérale a été nettement plus stricte dans une décision de 2015. Seul un danger concret pour la paix à l'école, et non un danger abstrait, donc purement théorique, pourrait justifier

26. BVerfGE 108, 282 (299 et s.), 24-09-2003. 
une telle interdiction ${ }^{27}$. Cette position s'explique vraisemblablement par le fait que, comme la Cour l'avait déjà souligné dans sa première décision, aucune plainte concrète à l'encontre du port de voile n'a été formulée de la part d'élèves ou de parents d'élèves. Et, selon les dires du ministère de la Hesse en charge des écoles, il n'y aurait eu effectivement aucun problème dans les établissements scolaires, même après cette décision.

Longtemps, les écolières qui refusaient de participer aux cours de natation pour des raisons religieuses pouvaient se prévaloir de la liberté de religion ${ }^{28}$. Ce n'est qu'avec l'arrivée des «burkinis ${ }^{29}$ que la situation a changé. Enfin, les tribunaux ont également dû se pencher sur les prières musulmanes d'un élève pendant les pauses dans une école dite «sensible». Le Tribunal administratif fédéral s'est prononcé pour l'interdiction, fondée sur un danger imminent pour la paix à l'école ${ }^{30}$.

\subsubsection{DANS LES ENCEINTES DE JUSTICE}

Vu de France, c'est une décision de la Cour constitutionnelle datant de 1972 qui retient l'attention dans le domaine de la justice ${ }^{31}$. Invoquant le Sermon sur la montagne, un pasteur protestant avait refusé de prêter le serment judiciaire prescrit par la loi. Dans ce sermon, on peut lire: "Que votre parole soit oui, oui ou non, non, ce qu'on y ajoute vient du malin ». La Cour constitutionnelle fédérale a validé ce refus. Le bon fonctionnement de la justice est certes un objectif à valeur constitutionnelle. Dans le cas présent toutefois, il était possible selon la Cour de ménager au profit du requérant une exception à l'obligation légale du serment. «L'affirmation de la vérité d'un témoignage, que le législateur tient pour indispensable à l'établissement de la vérité, ne doit pas nécessairement être faite sous la forme d'un serment comportant le mot "jurer" 32 ». Sur ce, le législateur a introduit une «affirmation solennelle» équivalente au serment.

De plus, certains Länder ont adopté ces dernières années des législations restreignant le port de symboles religieux, applicables principalement aux juges professionnels et dans certains cas également aux juges assesseurs.

27. BVerfGE 138, 296, 27-01-2015. V. A. GAILlEt, «Port du voile par les enseignantes des écoles publiques: retour à Karlsruhe», AJDA 2015, p. 1401.

28. BVerwGE 94, 82 (92 et s.), 25-08-1993.

29. BVerwGE 147, 362 (374 et s.), 11-09-2013.

30. BVerwG: JZ 2012, p. 358, note C. Enders.

31. BVerfGE 33, 23.

32. BVerfGE 33, 23 (32 et s.). 
Un recours en inconstitutionnalité, actuellement pendant devant la Cour constitutionnelle fédérale, permettra peut-être de clarifier le point de savoir si la neutralité de la justice justifie des règles spécifiques ${ }^{33}$.

\section{APPRÉCIATION}

La présentation qui précède démontre clairement qu'en Allemagne la liberté de religion ne peut pratiquement pas être limitée sur le fondement de «l'ordre public », à la différence de la France. Ce qui détermine l'approche de la liberté de religion, c'est une compréhension large de celle-ci, fondée sur le propre jugement de l'individu concerné quant à ce que signifie pour lui cette liberté, une compréhension large également de ce qui constitue une ingérence dans l'exercice de cette liberté ainsi que le niveau élevé des exigences susceptibles de justifier une telle ingérence. Il existe par conséquent très peu de décisions juridictionnelles qui valident des décisions étatiques interdisant un acte accompli ou à accomplir sur le fondement de la liberté de religion. La jurisprudence a longtemps porté sur des manifestations spécifiques du christianisme et elle se fondait notamment sur la dignité de la personne et son rapport étroit avec la liberté de religion ${ }^{34}$. Mais au cours des dernières années, elle a eu à s'intéresser à d'autres communautés religieuses comme l'islam.

Il existe bien entendu de nombreux débats sur le point de savoir si le champ de protection de la liberté de religion doit être préservé dans son ampleur, et si les obstacles à sa limitation doivent être maintenus au niveau élevé où la Cour constitutionnelle fédérale les a placés ${ }^{35}$. Jusqu'ici, aucune des tentatives visant à modifier la jurisprudence de la Cour constitutionnelle fédérale n'a été couronnée de succès. Une des principales explications pourrait être que jamais encore une situation ne s'est présentée dans laquelle les conflits sociaux liés à l'exercice de la liberté de religion se soient révélés insolubles.

33. 2 BvR 1333/17.

34. V. à ce sujet BVerfGE 32, 98 (106), 19-10-1971; 33, 23 (28 et s.), 11-04-1971.

35. Pour une critique de l'opinion majoritaire, V. par ex. K.-H. KÄsTnER, « Hypertrophie des Grundrechts auf Religionsfreiheit?», JZ 1998, p. 974; F. Sсносн, «Die Grundrechtsdogmatik vor den Herausforderungen einer multikulturellen Gesellschaft », in Festschrift Hollerbach, Berlin, Duncker \& Humblot, 2001, p. 149; U. VOSGERAU, Freiheit des Glaubens und Systematik des Grundgesetzes, Berlin, Duncker \& Humblot, 2007. 
D'un point de vue français, une telle réalité est assurément surprenante si l'on considère que ce pays a toujours été traversé par des conflits autour des activités religieuses, des controverses à propos des processions chrétiennes au début $\mathrm{du} \mathrm{xx}^{\mathrm{e}}$ siècle aux conflits concernant les crèches de Noël dans les mairies ou encore les tenues vestimentaires musulmanes - interdiction des signes religieux à l'école, port du voile par des accompagnatrices scolaires, interdiction de la dissimulation du visage (voile intégral), burkinis sur les plages...

Cette différence fondamentale s'explique peut-être par la relation qu'entretiennent l'État et la religion. En France, l'État et l'Église catholique se sont longtemps fait face, opposant ainsi deux forces dont les relations n'ont pas toujours été simples. L'Allemagne quant à elle connaît deux grandes confessions depuis le $\mathrm{XVI}^{\mathrm{e}}$ siècle. Même si dans les régions protestantes un système d'Église d'État s'est rapidement mis en place, l'État a dû faire preuve d'une certaine neutralité religieuse avec l'abandon du principe cuius regio eius religio au $\mathrm{XVI}^{\mathrm{e}}$ siècle.

Que ce soit pour cette raison ou pour d'autres, il reste que jamais les forces anticléricales ou antireligieuses n'ont atteint en Allemagne le niveau qui est le leur en France depuis le XIX siècle. Et au lendemain de la Seconde Guerre mondiale, la liberté de religion a joué un rôle encore plus important. Alors qu'après la période nazie l'autorité morale de l'État était fortement remise en cause, les Églises jouissaient d'un prestige incontesté. Cela s'est également reflété dans l'ordre juridique, et s'est traduit en particulier par l'importance attachée à l'autonomie des deux grandes Églises et au poids de la liberté individuelle de religion, en particulier au bénéfice des minorités - y compris des minorités chrétiennes.

L'émergence de l'islam depuis les années 1960 et 1970, liée pour l'essentiel à l'immigration turque, a certes changé la société. La propension à accepter les comportements déterminés par la religion est néanmoins largement répandue et en tout état de cause le système juridique n'a connu sur ce point aucun changement. 